\title{
Association between genetic risk variants and glucose intolerance during pregnancy in north Indian women
}

Geeti P. Arora ${ }^{1,2}$, Peter Almgren², Charlotte Brøns ${ }^{3}$, Richa G. Thaman', Allan A. Vaag ${ }^{2,3,5}$, Leif Groop 2,4 and Rashmi B. Prasad ${ }^{2^{*}}$

\begin{abstract}
Background: Gestational diabetes (GDM) is a more common problem in India than in many other parts of the world but it is not known whether this is due to unique environmental factors or a unique genetic background. To address this question we examined whether the same genetic variants associated with GDM and Type 2 Diabetes (T2D) in Caucasians also were associated with GDM in North Indian women.

Methods: Five thousand one hundred pregnant women of gestational age 24-28 weeks from Punjab were studied by a $75 \mathrm{~g}$ oral glucose tolerance test (OGTT). GDM was diagnosed by both WHO1999 and 2013 criteria. 79 single nucleotide polymorphisms (SNPs) previously associated with T2D and glycemic traits (12 of them also with GDM) and 6 SNPs from previous T2D associations based on Indian population (some also with European) were genotyped on a Sequenom platform or using Taqman assays in DNA from 4018 women.

Results: In support of previous findings in Caucasian GDM, SNPS at KCJN11 and GRB14 loci were nominally associated with GDM1999 risk in Indian women (both $p=0.02$ ). Notably, T2D risk alleles of the variant rs 1552224 near CENTD2, rs11708067 in ADCY5 and rs11605924 in CRY2 genes associated with protection from GDM regardless of criteria applied $(p<0.025)$. SNPs rs7607980 near COBLL1 $(p=0.0001)$, rs13389219 near GRB14 $(p=0.026)$ and rs 10423928 in the GIPR gene ( $p=0.012$ ) as well as the genetic risk score (GRS) for these previously shown insulin resistance loci here associated with insulin resistance defined by HOMA2-IR and showed a trend towards GDM. GRS comprised of 3 insulin secretion loci here associated with insulin secretion but not GDM.

Conclusions: GDM in women from Punjab in Northern India shows a genetic component, seemingly driven by insulin resistance and secretion and partly shared with GDM in other parts of the world. Most previous T2D loci discovered in European studies did not associate with GDM in North India, indicative of different genetic etiology or alternately, differences in the linkage disequilibrium (LD) structure between populations in which the associated SNPs were identified and Northern Indian women. Interestingly some T2D risk variants were in fact indicative of being protective for GDM in these Indian women.
\end{abstract}

Keywords: Genetics, Risk variant, Gestational diabetes mellitus, Single nucleotide polymorphism, Diagnostic criteria, Insulin resistance, Insulin secretion, Type 2 diabetes mellitus

\footnotetext{
* Correspondence: rashmi.prasad@med.lu.se

2Department of Clinical Sciences, Clinical Research Centre, Lund University,

Malmö, Sweden

Full list of author information is available at the end of the article
}

(c) The Author(s). 2018 Open Access This article is distributed under the terms of the Creative Commons Attribution 4.0 International License (http://creativecommons.org/licenses/by/4.0/), which permits unrestricted use, distribution, and reproduction in any medium, provided you give appropriate credit to the original author(s) and the source, provide a link to the Creative Commons license, and indicate if changes were made. The Creative Commons Public Domain Dedication waiver (http://creativecommons.org/publicdomain/zero/1.0/) applies to the data made available in this article, unless otherwise stated. 


\section{Background}

Gestational Diabetes Mellitus (GDM) has been officially defined as "carbohydrate intolerance" of variable severity with onset or first recognition during pregnancy [1-3] irrespective of treatment and whether or not the condition persists after pregnancy. GDM represents almost $90 \%$ of all pregnancies complicated by diabetes [4]. The prevalence of GDM is rapidly increasing, ranging from 2 to $14 \%$ depending upon diagnostic criteria $[5,6]$. In a study of South Indian women, GDM prevalence varied between 12 and 21\% [7] while another study of North Indian women reported a prevalence of $10 \%$ using WHO criteria [8]. The hallmark of GDM is increased insulin resistance accompanied by decreased compensatory insulin secretory response. Type 2 diabetes (T2D) is also caused by increased insulin resistance and decreased insulin secretion to compensate for the former. Thus, both T2D and GDM share the same pathophysiology which is influenced by similar risk factors like high body mass index (BMI), history of abnormal glucose intolerance, family history of diabetes, age, and ethnicity [9-11].

A family history of both T2D and GDM is known to increase GDM risk, indicative of a common genetic component underlying both T2D and GDM $[12,13]$. Till date, more than 120 T2D risk loci have been confirmed to be associated with T2D [14]. A large proportion of them have also shown association with GDM. T2D risk variants at the MTNR1B, FTO, TLE1, G6PC2, GCKR, TCF7L2, ADCY5, CDKAL1, TCF2, HNF1B, PPARG, $K C N J 11, S L C 30 A 8$ loci have previously been associated with GDM in European populations [15-18] whereas variants in the CDKAL1,CDKN2A/2B, MTNR1B and $K C N Q 1$ loci were associated with GDM in Korean women $[19,20]$.

Some genetic variants are more unique to Indian T2D patients e.g. the SGCG (rs9552911) and TMEM163 (rs998451) variants [21-25]. However, genetic studies of GDM in India are scarce. The SNPs rs7754840 and rs7756992 in the CDKAL1 gene were associated with GDM in South Indian women [26], while variants in the HMG20A (rs7178572) and HNF4A (rs4812829) genes were associated with both GDM and T2D [27]. The aim of the present study was to investigate whether a panel of known variants previously associated with GDM and T2D in Indian and European populations are associated with GDM in Punjabi women.

\section{Methods}

\section{Study population and phenotyping}

Five thousand one hundred pregnant women were recruited by applying a multistage random screening in the State of Punjab in North India for GDM. Pregnant women at gestational week 24-28 were randomly selected and recruited [8, 28]. This was part of a WDF supported project titled "Gestational diabetes in Punjab" with the goal to create and implement sustainable awareness, education, screening, intervention and treatment capacities of diabetes in pregnancy (GDM) within the public and private health care system, as well as in the general population in Punjab. The team included a chief research coordinator, an assistant coordinator, doctors, nurses, lab technicians from all selected sites both in private hospitals and public healthcare system. Approval for screening was obtained from DRME, Chandigarh, India. The recruitment sites included Recruitment sites:, Deep Hospital, Model Town, Ludhiana as the epicenter, Shri Rama Charitable Hospital, Ludhiana, Chawla Hospital, Ludhiana, Iqbal Hospital, Ludhiana, Government Medical Colleges and Hospital, Patiala, Amritsar and Faridkot, PHC Verka, Amritsar, Health Centre Bhadsoan, Patiala, Health Centre Faridkot. The project was approved by Independent ethics committee, Ludhiana in 2009. The ethics committee is registered with Office of Drugs Controller General (India) Directorate General of Health Services with Registration no. ECR/ 525/Inst/PB/2014.

Information was obtained on age, BMI, family history of diabetes, diet, habitat (urban or rural), education and religion. All information material and written consent forms were provided in 3 languages (Hindi, Punjabi \& English) and duly signed by the participants. The study protocol was approved by local Ethical Committees. Glucose was measured in venous plasma samples at fasting and at $2 \mathrm{~h}$ after a 75 g glucose challenge using glucometers (Accucheck-Roche Diagnostics). Fasting insulin concentrations were determined with ELISA (Diametra, Milan, Italy; intra- and inter-assay variation of $<5.0$ and $<10.0 \%$, respectively). The homeostatic model assessment (HOMA2) was used to quantify insulin resistance (HOMA2-IR) and beta-cell function (HOMA2-B) from fasting insulin and glucose values using the HOMA2 calculator v2.2.3 (http://https://www.dtu.ox.ac.uk/homacalculator/) [29]. GDM was diagnosed according to the WHO1999 (FPG $\geq 7.0 \mathrm{mmol} / \mathrm{l}$ and/or 2-h glucose $\geq 7.8 \mathrm{mmol} / \mathrm{l}$ ) and the adapted WHO2013 (FPG $\geq 5.1$ and/or 2-h glucose $\geq 8.5 \mathrm{mmol} / \mathrm{l}$ ) criteria (ref). The clinical characteristics of subjects are shown in Table 1.

\section{Genotyping}

DNA was extracted from frozen and stored buffy coats using (QIAGEN Autopure LS kits. Six SNPs previously associated with GDM or T2D in India [21, 22, 26, 27, 30] (Additional file 2: Table S1) and 79 SNPs previously associated with T2D in Europe and elsewhere from GWAS studies up to 2012 (some of these also with GDM risk from candidate gene studies in GDM populations) were genotyped in the present study (Additional file 2: Table 
Table 1 Study population characteristics

\begin{tabular}{|c|c|c|c|c|c|c|c|c|c|c|c|c|c|c|c|}
\hline & \multirow[b]{2}{*}{$\mathrm{N}$} & \multirow[b]{2}{*}{ Mean } & \multirow[b]{2}{*}{$\pm \mathrm{SD}$} & \multicolumn{3}{|c|}{ GDM1999 } & \multicolumn{3}{|c|}{ Controls } & \multicolumn{3}{|c|}{ GDM2013 } & \multicolumn{3}{|c|}{ Controls } \\
\hline & & & & $\mathrm{N}$ & Mean & $\pm \mathrm{SD}$ & $\mathrm{N}$ & Mean & $\pm \mathrm{SD}$ & $\mathrm{N}$ & Mean & $\pm \mathrm{SD}$ & $\mathrm{N}$ & Mean & $\pm \mathrm{SD}$ \\
\hline Age (years) & 4018 & 21.41 & 3.40 & 346 & 21.11 & 3.59 & 3672 & 21.44 & 3.38 & 1386 & 21.68 & 3.5 & 2632 & 21.27 & 3.34 \\
\hline BMl & 4018 & 24.11 & 4.34 & 346 & 24.28 & 4.71 & 3672 & 24.09 & 4.30 & 1386 & 24.36 & 4.48 & 2632 & 23.97 & 4.25 \\
\hline Fasting plasma glucose (mmol/l) & 4018 & 4.81 & 0.76 & 346 & 5.53 & 1.32 & 3672 & 4.74 & 0.65 & 1386 & 5.51 & 0.69 & 2632 & 4.44 & 0.49 \\
\hline Plasma insulin (pmol) & 4018 & 54.25 & 61.86 & 346 & 46.73 & 42.24 & 3672 & 54.96 & 63.35 & 1386 & 52.74 & 54.44 & 2632 & 55.05 & 65.43 \\
\hline 2 h glucose (venous, mmol/l) & 4018 & 6.20 & 1.37 & 346 & 9.15 & 1.83 & 3672 & 5.93 & 0.92 & 1386 & 6.85 & 1.70 & 2632 & 5.86 & 1.00 \\
\hline $\begin{array}{l}\text { homa2_b with steady state } \\
\text { glucose and insulin values }\end{array}$ & 3680 & 104.02 & 55.71 & 346 & 78.01 & 37.56 & 3672 & 106.36 & 56.49 & 1386 & 77.37 & 38.02 & 2632 & 117.92 & 58.36 \\
\hline $\begin{array}{l}\text { homa2_ir with steady state } \\
\text { glucose and insulin values }\end{array}$ & 3680 & 0.97 & 0.74 & 346 & 0.96 & 0.73 & 3672 & 0.97 & 0.74 & 1386 & 1.02 & 0.79 & 2632 & 0.95 & 0.71 \\
\hline
\end{tabular}

S1) [14] on a Sequenom Mass ARRAY Platform (Sequenom San Diego, CA, USA) PLEX using MALDI-TOF mass spectrometer [31] or Taqman allelic discrimination assays using an ABI Prism 7900 sequence detection system (Applied Biosystems, Foster City, CA, USA). Genotyping was performed at the Lund University Diabetes Centre, Sweden after obtaining permission from ICMR (dated 21 october 2010 and Office of Drugs Controller General (India)(dated 14/12/2010).

Replication genotyping of $6 \%$ of the samples showed > 98\% concordance. rs6467136, and rs7202877 had a Hardy-Weinberg equilibrium (HWE) $p$-value of $<0.001$ in unaffected women based on WHO1999 criteria and < 0.05 in unaffected women based on WHO2013 criteria and were hence removed from the analysis.

\section{Statistical analyses}

Association of selected SNPs with risk of GDM was assessed by logistic regression analysis adjusted for maternal age and BMI and results presented as ORs with 95\% confidence intervals (CI). We also tested for associations with fasting and 2-h glucose values as well as with fasting insulin and HOMA2-B and HOMA2-IR (Additional file 2: Table S1) using linear regression analysis with maternal age and BMI as covariates. Individuals with missing data were excluded. Data were logarithmically transformed before analysis. The power to detect association with GDM2013 including 1386 GDM women and 2632 controls at $p<0.0006(0.05 / 79)$ (after Bonferroni correction) for a SNP allele frequency of 0.3 and effect size 1.3 was 0.97 , which decreased to 0.64 for effect size 1.2 under an additive model. For GDM1999, with 346 GDM and 3672 controls, the corresponding figures were 0.39 and 0.12 respectively. For association with quantitative glucose traits, power to detect association was 1 at alpha 0.05 for and allele frequency of $0.3[32,33]$. A $p$-value of $\leq 0.05$ was considered statistically significant on account of the current analyses being replication of previously published associations.

Genetic risk scores for insulin secretion (HOMA-2B) and insulin resistance (HOMA-2IR) were calculated using SNPs previously associated with insulin secretion and insulin resistance. SNPs were assessed for linkage disequilibrium (LD) and for those in high $\mathrm{LD}\left(\mathrm{r}^{2}\right)$, only one representative SNP was retained. Individual scores were calculated based on number of risk alleles weighed by their effect sizes reported in previous GWAS studies and logistic regression was performed against normalized measures of insulin secretion and insulin resistance.

All calculations were implemented in STATA, plink 1.09 and SPSS v22.0.

\section{Results}

Among the 4018 genotyped women, applying the WHO2013 criteria resulted in a total of 1386 women with GDM (34.5\%) whereas the number was reduced to 346 (8.6\%) when WHO1999 criteria were used. Notably, only 283 (7.0\%) women were diagnosed using both GDM 2013 and GDM 1999 criteria (Additional file 1: Figure S1) [34]. This is concordant with our previously published reports on the larger subset of the same population comprising 5100 women [28]. HOMA2-B was lower in GDM women defined by both criteria compared to pregnant normal glucose tolerant women (PNGT). HOMA2-IR was also higher in women with GDM2013 who thereby were more insulin resistant than PNGT (Table 1).

\section{SNPs previously associated with GDM/T2D in India}

None of the 8 SNPs previously associated with GDM or T2D in Indian populations was here associated with GDM (Table 2). However, analysis for association with GDM1999 or GDM 2013 against controls who did not satisfy either criterion revealed the nominal association of rs7756992 in CDKAL1 while rs689 in INS showed a trend towards association with GDM2013 (Table 3). 
Table 2 Association of previously reported GDM and T2D loci from Indian population based studies with risk of GDM according to both criteria

\begin{tabular}{|c|c|c|c|c|c|c|c|c|c|c|c|c|c|}
\hline Genotype & EA & Chr & $\begin{array}{l}\text { Gene/nearest } \\
\text { gene }\end{array}$ & Location & OR_WHO1999 & $\begin{array}{l}\text { lower } \\
\mathrm{Cl}\end{array}$ & $\begin{array}{l}\text { upper } \\
\mathrm{Cl}\end{array}$ & P_who1999 & OR_WHO2013 & $\begin{array}{l}\text { lower } \\
\mathrm{Cl}\end{array}$ & $\begin{array}{l}\text { upper } \\
\mathrm{Cl}\end{array}$ & P_who2013 & $n$ \\
\hline rs998451 & $A$ & 2 & TMEM163 & intron & 0.987 & 0.795 & 1.224 & 0.902 & 0.959 & 0.843 & 1.09 & 0.518 & 3882 \\
\hline rs1799999 & A & 7 & PPPIR3A & missense & 0.862 & 0.728 & 1.02 & 0.083 & 0.997 & 0.905 & 1.098 & 0.953 & 3890 \\
\hline rs689 & A & 11 & INS & $5^{\prime} U T R$ & 1.077 & 0.879 & 1.319 & 0.474 & 1.033 & 0.914 & 1.167 & 0.603 & 3903 \\
\hline rs9552911 & A & 13 & SGCG & intron & 1.057 & 0.83 & 1.347 & 0.653 & 1.017 & 0.875 & 1.183 & 0.824 & 3890 \\
\hline rs4812829 & A & 20 & HNF4A & intron & 1.04 & 0.871 & 1.24 & 0.667 & 0.988 & 0.89 & 1.096 & 0.814 & 3801 \\
\hline rs7178572 & G & 15 & HMG20A & intron & 0.988 & 0.832 & 1.173 & 0.891 & 1.017 & 0.921 & 1.122 & 0.743 & 3541 \\
\hline rs7756992 & G & 6 & CDKAL 1 & intron & 0.91 & 0.75 & 1.1 & 0.34 & 0.97 & 0.87 & 1.08 & 0.64 & 3686 \\
\hline rs7754840 & C & 6 & CDKAL1 & intron & 0.87 & 0.72 & 1.06 & 0.17 & 0.96 & 0.86 & 1.07 & 0.51 & 3721 \\
\hline
\end{tabular}

EA effect allele, OR_WHO1999 odds ratio based on WHO1999 criteria, OR_WHO2013 Odds ratio based on WHO2013 criteria, Cl confidence interval

\section{Previously reported GDM risk loci}

Out of 12 selected previously reported GDM risk loci, the $\mathrm{T}$ allele of the missense SNP rs5219 in the KCNJ11 gene was nominally associated with GDM1999 ( $p=$ 0.019) (Table 4). Contrary to previous reports, the risk allele A of SNP rs11708067 in the ADCY5 gene showed reduced risk for GDM defined by $2013(p=0.037)$ (Table 4) but not by 1999 criteria. The SNP rs2796441 in the TLE1 gene was associated with decreased insulin secretion $(p=$ 0.013) (Additional file 2: Table S2). The rs13266634 at SLC30A8 locus associated with GDM1999 while SNPs rs5219 in KCNJ11 and rs11708067 in ADCY5 associated with GDM2013 nominally when controls satisfying neither GDM diagnosis criteria were considered (Table 3).

\section{Previously reported T2D loci}

The risk allele C of SNP rs13389219 in the GRB14 gene was associated with GDM1999 $(p=0.022)$ (Table 5) but not with GDM2013 $(p=0.058)$ (Table 5). The T2D risk allele T of SNP rs11920090 in the intron of the SLC2A2 gene was associated with GDM2013 $(p=0.030)$ (Table 5).

Surprisingly, the T2D risk allele A of SNP rs11605924 in the $C R Y 2$ gene was associated with reduced risk of GDM1999 $(p=0.025)$ (Table 5). The same variant associated with GDM1999 in a sensitivity analysis when controls meeting neither GDM diagnosis criteria were considered (Table 3). In support of this, the same allele was also associated with lower 2-h glucose levels $(p=$ 0.038) (Additional file 2: Table S3).

Table 3 Association of previously reported GDM loci with risk of GDM according to both criteria

\begin{tabular}{|c|c|c|c|c|c|c|c|c|c|c|c|c|c|}
\hline \multirow[t]{2}{*}{ SNP } & \multirow[t]{2}{*}{ EA } & \multirow[t]{2}{*}{ Chr } & \multirow{2}{*}{$\begin{array}{l}\text { Gene/nearest } \\
\text { gene }\end{array}$} & \multirow[t]{2}{*}{ Location } & \multicolumn{4}{|c|}{ WHO 1999} & \multicolumn{4}{|c|}{ WHO 2013} & \multirow[t]{2}{*}{$\mathrm{n}$} \\
\hline & & & & & OR & Cl(lower) & Cl(upper) & $p$-value & OR & Cl(lower) & Cl(upper) & $p$-value & \\
\hline rs9939609 & A & 16 & FTO & intron & 1.04 & 0.86 & 1.26 & 0.67 & 0.98 & 0.88 & 1.10 & 0.83 & 3120 \\
\hline rs2796441 & G & 9 & TLE 1 & intergenic & 0.99 & 0.84 & 1.16 & 0.92 & 1.07 & 0.97 & 1.17 & 0.15 & 3905 \\
\hline rs560887 & C & 2 & $\mathrm{G} 6 \mathrm{PC} / \mathrm{ABCB} 11$ & intron & 1.18 & 0.92 & 1.52 & 0.19 & 1.11 & 0.96 & 1.28 & 0.13 & 3910 \\
\hline rs11708067 & A & 3 & $A D C Y 5$ & intron & 0.98 & 0.81 & 1.18 & 0.86 & 0.88 & 0.79 & 0.99 & 0.037 & 3877 \\
\hline rs1111875 & C & 10 & HHEX & intergenic & 0.90 & 0.77 & 1.06 & 0.22 & 1.05 & 0.96 & 1.16 & 0.24 & 3901 \\
\hline rs10811661 & $\mathrm{T}$ & 9 & CDKN2A $2 B$ & intergenic & 0.99 & 0.77 & 1.26 & 0.93 & 1.08 & 0.94 & 1.25 & 0.23 & 3890 \\
\hline rs4402960 & $\mathrm{T}$ & 3 & IGF2BP2 & intron & 1.02 & 0.87 & 1.20 & 0.77 & 0.95 & 0.86 & 1.04 & 0.29 & 3750 \\
\hline rs13266634 & C & 8 & SLC30A8 & coding-missense & 0.96 & 0.79 & 1.17 & 0.75 & 0.97 & 0.87 & 1.08 & 0.61 & 3898 \\
\hline rs7903146 & $\mathrm{T}$ & 10 & TCF7L2 & Intronic/promoter & 1.13 & 0.95 & 1.35 & 0.14 & 1.01 & 0.916 & 1.12 & 0.76 & 3543 \\
\hline rs10830963 & G & 11 & MTNRIB & intron & 0.89 & 0.75 & 1.05 & 0.20 & 0.98 & 0.89 & 1.08 & 0.69 & 3714 \\
\hline rs1801282 & C & 3 & PPARG & Coding-missense & 0.86 & 0.89 & 1.12 & 0.22 & 0.99 & 0.93 & 1.08 & 0.21 & 3652 \\
\hline rs10010131 & G & 4 & WFS1 & intron & 1.13 & 0.95 & 1.36 & 0.16 & 0.99 & 0.90 & 1.10 & 0.99 & 3843 \\
\hline rs5219 & $\mathrm{T}$ & 11 & KCNJ11 & coding-missense & 1.21 & 1.03 & 1.42 & 0.019 & 1.00 & 0.90 & 1.10 & 0.99 & 3595 \\
\hline
\end{tabular}

EA effect allele, OR_WHO1999 odds ratio based on WHO1999 criteria, OR_WHO2013 Odds ratio based on WHO2013 criteria, Cl confidence interval significant $p$ values where $p<0.05$ are indicated in bold 
Table 4 Association of previously reported T2D loci with risk of GDM according to both criteria

\begin{tabular}{|c|c|c|c|c|c|c|c|c|c|c|c|c|c|}
\hline \multirow[t]{2}{*}{ SNP } & \multirow[t]{2}{*}{ EA } & \multirow[t]{2}{*}{ Chr } & \multirow{2}{*}{$\begin{array}{l}\text { Gene/nearest } \\
\text { gene }\end{array}$} & \multirow[t]{2}{*}{ Location } & \multicolumn{4}{|c|}{ WHO 1999} & \multicolumn{4}{|c|}{ WHO 2013} & \multirow[t]{2}{*}{$n$} \\
\hline & & & & & $\mathrm{OR}$ & Cl(lower) & Cl(upper) & $p$-value & $\mathrm{OR}$ & Cl(lower) & $\mathrm{Cl}$ (upper) & $p$-value & \\
\hline rs2296172 & G & 1 & MACF1 & coding-missense & 0.92 & 0.71 & 1.20 & 0.56 & 1.04 & 0.89 & 1.21 & 0.58 & 3847 \\
\hline rs340874 & C & 1 & PROX1 & intergenic & 0.94 & 0.80 & 1.11 & 0.52 & 0.96 & 0.87 & 1.06 & 0.47 & 3709 \\
\hline rs7578597 & $\mathrm{T}$ & 2 & THADA & coding-missense & 0.90 & 0.72 & 1.12 & 0.37 & 0.92 & 0.80 & 1.06 & 0.27 & 3710 \\
\hline rs243088 & $\mathrm{T}$ & 2 & $B C L 11 A$ & intergenic & 1.10 & 0.94 & 1.29 & 0.22 & 1.07 & 0.97 & 1.18 & 0.15 & 3717 \\
\hline rs7593730 & $\mathrm{T}$ & 2 & RBMS1/ITGB6 & intronic & 1.01 & 0.84 & 1.22 & 0.83 & 0.99 & 0.88 & 1.11 & 0.93 & 3906 \\
\hline rs7607980 & C & 2 & COBLL1 & coding-missense & 0.95 & 0.73 & 1.24 & 0.75 & 0.95 & 0.81 & 1.11 & 0.52 & 3885 \\
\hline rs13389219 & C & 2 & GRB14 & intergenic & 1.25 & 1.03 & 1.52 & 0.022 & 1.11 & 0.99 & 1.23 & 0.058 & 3829 \\
\hline rs7578326 & A & 2 & KIAA1486/IRS1 & $\begin{array}{l}\text { intron of uncharacterized } \\
\text { LOC646736 }\end{array}$ & 0.97 & 0.80 & 1.18 & 0.78 & 0.98 & 0.87 & 1.10 & 0.79 & 3600 \\
\hline rs2943641 & C & 2 & IRSI & intergenic & 0.92 & 0.76 & 1.12 & 0.43 & 0.97 & 0.87 & 1.09 & 0.67 & 3643 \\
\hline rs4675095 & A & 2 & IRSI & intron & 1.11 & 0.87 & 1.42 & 0.39 & 1.04 & 0.90 & 1.19 & 0.58 & 3817 \\
\hline rs831571 & C & 3 & PSMD6 & intergenic & 1.02 & 0.84 & 1.25 & 0.77 & 0.93 & 0.83 & 1.05 & 0.26 & 3726 \\
\hline rs4607103 & C & 3 & ADAMTS9-AS2 & intron & 1.14 & 0.98 & 1.33 & 0.08 & 1.00 & 0.91 & 1.09 & 0.97 & 3884 \\
\hline rs11920090 & $\mathrm{T}$ & 3 & $S L C 2 A 2$ & intron & 1.19 & 0.93 & 1.51 & 0.16 & 1.16 & 1.01 & 1.33 & 0.03 & 3606 \\
\hline rs6815464 & C & 4 & MAEA & intron & 1.04 & 0.83 & 1.30 & 0.71 & 1.03 & 0.90 & 1.18 & 0.64 & 3722 \\
\hline rs459193 & G & 5 & ANKRD55 & intergenic & 0.99 & 0.84 & 1.16 & 0.90 & 1.07 & 0.97 & 1.18 & 0.16 & 3884 \\
\hline rs4457053 & G & 5 & ZBED3 & intron of ZBED3-AS1 & 1.05 & 0.86 & 1.29 & 0.57 & 0.95 & 0.84 & 1.07 & 0.45 & 3579 \\
\hline rs9470794 & C & 6 & ZFAND3 & intron & 1.07 & 0.85 & 1.35 & 0.51 & 1.05 & 0.91 & 1.21 & 0.48 & 3608 \\
\hline rs17168486 & $\mathrm{T}$ & 7 & $D G K B$ & intergenic & 0.99 & 0.83 & 1.17 & 0.92 & 0.97 & 0.88 & 1.07 & 0.62 & 3855 \\
\hline rs2191349 & $\mathrm{T}$ & 7 & DGKB/TMEM195 & intergenic & 1.04 & 0.88 & 1.22 & 0.62 & 1.00 & 0.91 & 1.10 & 0.95 & 3903 \\
\hline rs864745 & $\mathrm{T}$ & 7 & JAZF1 & intron & 0.98 & 0.83 & 1.16 & 0.87 & 1.02 & 0.92 & 1.13 & 0.68 & 3876 \\
\hline rs4607517 & A & 7 & GCK & intergenic & 1.04 & 0.82 & 1.32 & 0.70 & 1.01 & 0.88 & 1.16 & 0.86 & 3903 \\
\hline rs17133918 & C & 7 & GRB10 & intron & 1.03 & 0.87 & 1.23 & 0.67 & 0.97 & 0.88 & 1.08 & 0.65 & 3907 \\
\hline rs933360 & A & 7 & GRB10 & intron & 1.03 & 0.87 & 1.22 & 0.70 & 1.03 & 0.93 & 1.14 & 0.54 & 3905 \\
\hline rs6943153 & C & 7 & GRB10 & intron & 0.86 & 0.73 & 1.03 & 0.11 & 0.95 & 0.86 & 1.05 & 0.36 & 3602 \\
\hline rs516946 & C & 8 & ANK1 & intron & 1.01 & 0.82 & 1.23 & 0.91 & 1.09 & 0.97 & 1.23 & 0.13 & 3922 \\
\hline rs896854 & $\mathrm{T}$ & 8 & TP53INP1 & intron & 0.97 & 0.83 & 1.14 & 0.75 & 0.97 & 0.88 & 1.06 & 0.57 & 3903 \\
\hline rs7034200 & A & 9 & GLIS3 & intron & 0.98 & 0.83 & 1.15 & 0.84 & 1.03 & 0.93 & 1.13 & 0.52 & 3868 \\
\hline rs13292136 & C & 9 & TLE4 (CHCHD9) & intergenic & 0.94 & 0.75 & 1.18 & 0.62 & 0.98 & 0.86 & 1.12 & 0.79 & 3706 \\
\hline rs12571751 & A & 10 & $Z M I Z 1$ & intron & 0.86 & 0.73 & 1.01 & 0.07 & 0.96 & 0.87 & 1.06 & 0.49 & 3601 \\
\hline rs553668 & A & 10 & $A D R A 2 A$ & UTR-3 & 1.17 & 0.99 & 1.39 & 0.06 & 1.07 & 0.97 & 1.19 & 0.15 & 3666 \\
\hline rs10885122 & G & 10 & $A D R A 2 A$ & intergenic & 1.03 & 0.84 & 1.27 & 0.75 & 1.05 & 0.93 & 1.18 & 0.42 & 3683 \\
\hline rs163184 & G & 11 & KCNQ1 & intron & 0.90 & 0.76 & 1.07 & 0.23 & 1.00 & 0.90 & 1.10 & 0.98 & 3713 \\
\hline rs2237895 & C & 11 & KCNQ1 & intron & 0.96 & 0.81 & 1.13 & 0.66 & 1.01 & 0.92 & 1.11 & 0.79 & 3682 \\
\hline rs11605924 & A & 11 & $C R Y 2$ & intron & 0.84 & 0.72 & 0.97 & 0.025 & 1.00 & 0.92 & 1.10 & 0.85 & 3909 \\
\hline rs7944584 & A & 11 & MADD & intron & 0.91 & 0.74 & 1.13 & 0.41 & 1.09 & 0.96 & 1.23 & 0.15 & 3553 \\
\hline rs 174550 & $\mathrm{~T}$ & 11 & FADS1 & intron & 0.94 & 0.76 & 1.17 & 0.62 & 0.96 & 0.85 & 1.09 & 0.56 & 3908 \\
\hline rs1552224 & A & 11 & CENTD2 & intergenic & 0.92 & 0.75 & 1.13 & 0.45 & 0.81 & 0.72 & 0.92 & 0.001 & 3911 \\
\hline rs11063069 & G & 12 & CCND2 & intergenic & 0.99 & 0.80 & 1.23 & 0.98 & 1.04 & 0.91 & 1.19 & 0.52 & 3671 \\
\hline rs10842994 & C & 12 & KLHDC5 & intergenic & 1.13 & 0.89 & 1.44 & 0.28 & 0.97 & 0.84 & 1.11 & 0.67 & 3906 \\
\hline rs1153188 & A & 12 & $D C D$ & intergenic & 1.15 & 0.93 & 1.42 & 0.19 & 1.01 & 0.89 & 1.14 & 0.82 & 3912 \\
\hline rs1531343 & C & 12 & HMGA2 & intron of pseudogene & 0.83 & 0.67 & 1.03 & 0.09 & 0.90 & 0.80 & 1.02 & 0.10 & 3915 \\
\hline rs7961581 & C & 12 & TSPAN8,LGR5 & intergenic & 0.91 & 0.77 & 1.08 & 0.31 & 1.02 & 0.92 & 1.13 & 0.61 & 3703 \\
\hline
\end{tabular}


Table 4 Association of previously reported T2D loci with risk of GDM according to both criteria (Continued)

\begin{tabular}{|c|c|c|c|c|c|c|c|c|c|c|c|c|c|}
\hline \multirow[t]{2}{*}{ SNP } & \multirow[t]{2}{*}{ EA } & \multirow[t]{2}{*}{ Chr } & \multirow{2}{*}{$\begin{array}{l}\text { Gene/nearest } \\
\text { gene }\end{array}$} & \multirow[t]{2}{*}{ Location } & \multicolumn{4}{|c|}{ WHO 1999} & \multicolumn{4}{|c|}{ WHO 2013} & \multirow[t]{2}{*}{$n$} \\
\hline & & & & & $\mathrm{OR}$ & Cl(lower) & Cl(upper) & $p$-value & $\mathrm{OR}$ & $\mathrm{Cl}($ lower) & Cl(upper) & $p$-value & \\
\hline rs7957197 & $\mathrm{T}$ & 12 & OASL/TCF1/HNF1A & intron of QASL & 0.87 & 0.65 & 1.17 & 0.37 & 1.00 & 0.83 & 1.21 & 0.96 & 3924 \\
\hline rs17271305 & G & 15 & VPS13C & intron & 1.02 & 0.86 & 1.20 & 0.81 & 0.92 & 0.83 & 1.02 & 0.15 & 3825 \\
\hline rs11071657 & $A$ & 15 & FAM148B & intergenic & 1.03 & 0.87 & 1.22 & 0.72 & 0.92 & 0.83 & 1.02 & 0.13 & 3897 \\
\hline rs7177055 & A & 15 & HMG2OA & intergenic & 1.00 & 0.85 & 1.17 & 0.99 & 0.98 & 0.89 & 1.08 & 0.74 & 3907 \\
\hline rs35767 & G & 12 & IGF1 & nearGene-5 & 0.88 & 0.91 & 1.10 & 0.19 & 0.93 & 0.94 & 1.06 & 0.21 & 3910 \\
\hline rs11634397 & G & 15 & ZFAND6 & intergenic & 0.89 & 0.76 & 1.04 & 0.16 & 0.96 & 0.87 & 1.06 & 0.47 & 3910 \\
\hline rs8042680 & A & 15 & $P R C 1$ & intron & 0.89 & 0.76 & 1.04 & 0.16 & 0.99 & 0.90 & 1.10 & 0.95 & 3887 \\
\hline rs8090011 & G & 18 & LAMA1 & intron & 0.95 & 0.81 & 1.11 & 0.57 & 0.93 & 0.84 & 1.02 & 0.13 & 3911 \\
\hline rs10401969 & $C$ & 19 & SUGP1 & intron & 0.96 & 0.72 & 1.27 & 0.79 & 0.86 & 0.72 & 1.01 & 0.07 & 3605 \\
\hline rs8108269 & G & 19 & GIPR & intergenic & 1.02 & 0.85 & 1.23 & 0.77 & 1.07 & 0.96 & 1.19 & 0.16 & 3508 \\
\hline rs10423928 & A & 19 & GIPR & intron & 0.85 & 0.67 & 1.08 & 0.20 & 1.06 & 0.93 & 1.20 & 0.37 & 3911 \\
\hline rs6017317 & G & 20 & $\begin{array}{l}\text { FITM2-R3HDML- } \\
\text { HNF4A }\end{array}$ & intergenic & 0.96 & 0.81 & 1.13 & 0.64 & 0.98 & 0.89 & 1.08 & 0.72 & 3758 \\
\hline rs5945326 & A & $x$ & DUSP9 & intergenic & 0.95 & 0.81 & 1.12 & 0.58 & 1.01 & 0.92 & 1.12 & 0.74 & 3589 \\
\hline
\end{tabular}

EA effect allele, OR_WHO1999 odds ratio based on WHO1999 criteria, OR_WHO2013 Odds ratio based on WHO2013 criteria, CI confidence interval significant $p$ values where $p<0.05$ are indicated in bold

The risk allele A of SNP rs1552224 in the CENTD2 locus was associated with decreased risk of GDM2013 $(p=0.001)$ (Table 5).

\section{Association with insulin secretion and insulin resistance}

Twelve SNPs previously associated with insulin secretion were here tested for association with HOMA2-B. The T2D risk allele A of rs11071657 at the FAM148B locus was nominally associated with increased insulin secretion $(p=0.044)$ (Table 6). A GRS comprising of 3 previously reported insulin secretion loci with the lowest $p$-values for insulin secretion in the present study associated with insulin secretion in the present study $(p=0.008$, beta $=0.25$, $\mathrm{SE}=0.098)$. GRS for insulin secretion did not associate with either GDM2013 $(p=0.15$, beta $=-0.06, \mathrm{SE}=0.045)$ or $\operatorname{GDM} 1999(p=0.73$, beta $=-0.009, \mathrm{SE}=0.026)$.

Of 6 SNPs previously associated with measures of insulin resistance, 3 SNPs here associated with HOMA2-IR. The $\mathrm{C}$ allele of rs7607980 in the COBLL1 gene was associated with decreased HOMA2-IR $(p=$ $0.0001)$. The $C$ allele of rs13389219 near GRB14 ( $p=$ $0.026)$ and A allele of rs10423928 in the intron of the GIPR gene $(p=0.012)$ showed worse insulin resistance (increased HOMA2-IR; Table 7). Genetic risk scores (GRS) calculated based on the 3 SNPs associated with insulin resistance showed an increase of insulin resistance by $0.07(\mathrm{SE}=0.145, p=0.006)$ per allele. GRS for insulin resistance showed a trend towards GDM2013

Table 5 Sensitivity analysis for association of selected risk variants with GDM risk

\begin{tabular}{|c|c|c|c|c|c|c|c|c|c|c|c|c|c|c|}
\hline \multirow[t]{2}{*}{ SNP } & \multirow[t]{2}{*}{ EA } & \multirow[t]{2}{*}{ Chr } & \multirow{2}{*}{$\begin{array}{l}\text { Gene/ } \\
\text { nearest } \\
\text { gene }\end{array}$} & \multirow[t]{2}{*}{ Location } & \multicolumn{5}{|c|}{ WHO 1999} & \multicolumn{4}{|c|}{ WHO 2013} & \multirow[t]{2}{*}{$n$} \\
\hline & & & & & OR & Cl(lower) & Cl(upper) & $p$-value & $n$ & OR & Cl(lower) & Cl(upper) & $p$-value & \\
\hline rs13266634 & $T$ & 8 & SLC30A8 & coding-missense & 1.24 & 1.01 & 1.53 & 0.037 & 2834 & 1.049 & 0.91 & 1.21 & 0.50 & 383 \\
\hline rs11605924 & A & 11 & CRY2 & intron & 0.84 & 0.71 & 0.99 & 0.038 & 2833 & 1.005 & 0.91 & 1.10 & 0.91 & 384 \\
\hline rs35767 & $\mathrm{T}$ & 12 & $|G F|$ & nearGene-5 & 1.26 & 1.00 & 1.60 & 0.054 & 2837 & 1.15 & 0.98 & 1.33 & 0.07 & 384 \\
\hline rs5219 & $\mathrm{T}$ & 11 & KCNJ11 & coding -missense & 1.18 & 1.00 & 1.40 & 0.059 & 2605 & 1.00 & 0.91 & 1.11 & 0.91 & טכו \\
\hline rs11708067 & G & 3 & $A D C Y 5$ & intron & 1.11 & 0.86 & 1.44 & 0.42 & 2810 & 1.25 & 1.09 & 1.45 & 0.002 & 381 \\
\hline rs689a & A & 11 & INS & Promoter/intron & 0.91 & 0.64 & 1.29 & 0.60 & 2835 & 0.81 & 0.65 & 1.00 & 0.054 & $3 \varepsilon$ \\
\hline rs8108269 & G & 19 & GIPR & intergenic & 1.14 & 0.94 & 1.36 & 0.17 & 2568 & 1.12 & 0.99 & 1.25 & 0.059 & 34 \\
\hline rs7756992 ${ }^{a}$ & G & 6 & CDKAL1 & intron & 0.96 & 0.76 & 1.19 & 0.69 & 2670 & 2.80 & 1.00 & 7.87 & 0.049 & 36 \\
\hline
\end{tabular}

aindicates loci previously associated with GDM / T2D in India or GDM in studies based on the European population

Logistic regression was performed on GDM cases diagnosed according to WHO1999 and WHO2013 criteria against controls who had no GDM diagnosis using either criteria

significant $p$ values where $p<0.05$ are indicated in bold 
Table 6 Association of selected loci with insulin secretion (HOMA2-B)

\begin{tabular}{|c|c|c|c|c|c|c|c|c|}
\hline SNP & EA & Chr & Gene/nearest gene & Location & Beta & SE & $p$-value & $N$ \\
\hline rs340874 & $C$ & 1 & PROX1 & intergenic & 0.009 & 0.011 & 0.388 & 3395 \\
\hline rs560887 & $C$ & 2 & $\mathrm{G} 6 \mathrm{PC} 2 / A B C B 11$ & intron & -0.004 & 0.016 & 0.818 & 3578 \\
\hline rs11708067 & A & 3 & $A D C Y 5$ & intron & -0.024 & 0.012 & 0.053 & 3556 \\
\hline rs11920090 & $\mathrm{T}$ & 3 & $S L C 2 A 2$ & intron & -0.014 & 0.015 & 0.361 & 3301 \\
\hline rs4607517 & A & 7 & GCK & intergenic & 0.007 & 0.012 & 0.571 & 3372 \\
\hline rs2191349 & $\mathrm{T}$ & 7 & DGKB/TMEM195 & intergenic & -0.008 & 0.011 & 0.480 & 3575 \\
\hline rs7034200 & A & 9 & GLIS3 & intron & 0.002 & 0.016 & 0.922 & 3576 \\
\hline rs10885122 & G & 10 & ADRA2A & intergenic & -0.006 & 0.010 & 0.546 & 3545 \\
\hline rs7944584 & $A$ & 11 & MADD & intron & -0.021 & 0.013 & 0.116 & 3372 \\
\hline rs7903146 & $\mathrm{T}$ & 10 & TCF7L2 & Intronic/promoter & 0.003 & 0.011 & 0.798 & 3240 \\
\hline rs10830963 & G & 11 & MTNR1B & intron & -0.007 & 0.011 & 0.473 & 3398 \\
\hline rs 174550 & $\mathrm{~T}$ & 11 & FADS1 & intron & 0.011 & 0.014 & 0.435 & 3248 \\
\hline rs7756992 & G & 6 & CDKAL1 & intron & 0.011 & 0.014 & 0.446 & 3576 \\
\hline rs11071657 & $A$ & 15 & FAM148B & intergenic & -0.023 & 0.011 & 0.044 & 3568 \\
\hline
\end{tabular}

significant $p$ values where $p<0.05$ are indicated in bold

$(p=0.065$, beta $=0.076, \mathrm{SE}=0.04)$ but not GDM1999

$(p=0.14$, beta $=0.023, \mathrm{SE}=0.025)$.

\section{Discussion}

In this large study, we investigated the genetic basis of gestational diabetes mellitus in Punjabi Indian women $[15,16,19,27]$.

Surprisingly, the genetic variants in the HMG20A and HNF4A genes which previously have been associated with risk of T2D and GDM in South India [27] were not associated with GDM or T2D in Punjabi pregnant women. This could be due to differences in allele frequencies between the North and South Indian populations, which are ethnically quite distinctive populations [35]. The Punjabi Indian population belongs to the "Ancestral North Indians" group and shares genetic similarities with populations from Middle East, Central Asia and to some degree Europe whereas the South Indian population genetically belongs to the distinct "Ancestral South Indian" group [35]. Notably the CDKAL1 variant associated with GDM only when a sensitivity analysis was performed using controls that had no GDM diagnosis using either GDM1999 or GDM2013 criteria, thus replicating a previous association.

Neither did we observe associations with loci associated with GDM elsewhere including variants in the CDKAL1 and MTNR1B loci, which have been reported to be associated with GDM in South Korea [19]. A sensitivity analysis using controls that had no GDM diagnosis using either criterion revealed the nominal association of variants in SLC30A8, KCNJ11 and ADCY5. These largely negative findings could be attributed to population-based differences. Previous studies have indicated differences in anthropometry between Indian and
European populations, with the former manifesting a "thin-fat" phenotype [36]. Subsequently, it is possible that since most T2D loci were identified in European ancestry cohorts, the negative findings could reflect differences in tagging SNPs due to differences haplotypes between populations. On the other hand, the underlying etiology of GDM could also be different genetically. While the study population is the largest GDM study till date, this might lack sufficient power to detect genome-wide significance levels of association with an unstable phenotype. The effect sizes of previously reported T2D loci were low, generally under odds ratios of 1.2 , therefore the study was not sufficiently powered to demonstrate association of SNPs with such low effect sizes. Alternately, considering the lack of consensus for GDM diagnosis criteria worldwide, it is plausible that this could be due to different thresholds that might apply for the Indian population.

Notably, T2D risk variants in the CRY2 (WHO1999), CENTD2 (WHO2013) and the ADCY5 (WHO2013) genes were here protective for GDM. CRY2 encodes for the cryptochrome protein involved in the regulation of the circadian clock. Risk allele carriers of the rs11708067 SNP in $A D C Y 5$ has previously been shown to reduce ADCY5 expression in pancreatic beta cells and important for coupling glucose to insulin secretion in human islets [37]. It has been previously shown that T2D risk alleles show extreme directional differentiation across various populations, with T2D risk alleles decreasing in frequency along human migration into East Asia [38]. Such flip-flops of risk alleles may be explained by population differences, possibly due to genetics or environment. Alternately, such "flip-flop" associations have also been attributed to multi-locus effects as shown from 
Table 7 Association with HOMA-IR selected loci: insulin resistance SNPs

\begin{tabular}{|c|c|c|c|c|c|c|c|c|}
\hline SNP & EA & Chr & Gene/nearest gene & Location & Beta & SE & $p$-value & $\mathrm{N}$ \\
\hline rs2943641 & C & 2 & IRSI & intergenic & -0.001 & 0.014 & 0.923 & 3337 \\
\hline rs4675095 & A & 2 & IRSI & intron & -0.028 & 0.017 & 0.102 & 3500 \\
\hline rs4607517 & A & 7 & GCK & intergenic & 0.018 & 0.018 & 0.299 & 3576 \\
\hline rs7607980 & C & 2 & COBLL1 & coding-missense & 0.070 & 0.019 & 0.0001 & 3557 \\
\hline rs13389219 & C & 2 & GRB14 & intergenic & 0.029 & 0.013 & 0.026 & 3518 \\
\hline rs10423928 & A & 19 & GIPR & intron & 0.041 & 0.016 & 0.012 & 3585 \\
\hline
\end{tabular}

significant $p$ values where $p<0.05$ are indicated in bold

theoretical modeling studies demonstrating that the direction of allelic effect may flip when tested allele is inversely correlated with another risk allele at another locus, or positively correlated with a protective allele at another locus [39].

A HWE threshold of $<0.001$ in unaffected individuals based in either criteria was set as a cut-off; SNPs showing significant deviations from HWE should be interpreted with caution, since these could be indicative of population substructures, inbreeding or selection. The current study only comprises genotyping data from candidate SNPs which do not provide sufficient coverage of the genome to detail population stratification or inbreeding. HWE could also be indicative of actual association. A serious problem in the study of the genetics of GDM is the implementation of different criteria, since some women could be classified as controls based on different criteria. For SNP rs5219 in KCNJ11 (HWE $p=$ 0.004, WHO1999; HWE $p=0.01$, WHO2013) and rs11605924 in CRY2 (HWE $p=0.007$ WHO1999 and HWE $p=0.06$, WHO2013), HWE values were nominally significant for the same criteria where an association was observed; these findings need to be replicated in independent cohorts.

Of 6 loci previously associated with insulin resistance, here 3 also showed an association with HOMA2-IR and a trend towards significance for GDM2013 but not GDM1999 including SNPs rs7607980 in the COBLL1 gene [40], rs13389219 near GRB14 and rs10423928 in the GIPR gene indicating that some of the genetic basis seem to be driven by previously reported insulin resistance loci. Similarly, a GRS with the 3 variants with the lowest $p$-values for insulin secretion associated with insulin secretion but not GDM2013 or GDM1999.

Taken together, the results demonstrate that GDM in women from Punjab in Northern India shows a genetic component, partially shared with GDM in other parts of the world, and seems to be driven by both insulin resistance and secretion. However, the direction of the effect can differ; some T2D risk variants were indicative of being protective for GDM in these Indian women.

\section{Conclusions}

GDM in women from Punjab in Northern India shows a genetic component shared with T2D. This genetic basis is seemingly driven by a complex interplay between insulin secretion and sensitivity during pregnancy and is at least partly shared with GDM in other parts of the world. Interestingly some of the T2D risk variants in $A D C Y 5$ and $C R Y 2$ were protective against GDM. Most of the previous T2D loci discovered in European studies did not associate with GDM in North India. Interestingly some T2D risk variants were in fact indicative of being protective for GDM in these Indian women. This could be attributed to different genetic etiology or differences in the LD structure between populations in which the associated SNPs were identified and Northern Indian women. GWAS or whole genome sequencing will be interesting to further unravel the genetic basis of GDM in India.

\section{Additional file}

Additional file 1: Figure S1. Number of GDM women according to WHO2013 and WHO1999 criteria. (PDF 81 kb)

Additional file 2: Table S1. T2D M associated SNPS selected from previously published GWAS studies upto 2012 and GDM associated loci from previous candidate and GWAS studies. (*) indicate SNPS previously associated with GDM or T2D in India. Table S2. Association of previously reported GDM loci with glycemic traits. Table S3. Association of GDM loci identified in the current study with glycemic traits. (XLSX $30 \mathrm{~kb}$ )

\footnotetext{
Abbreviations

GDM: Gestational diabetes mellitus; GRS: Genetic risk score; GWAS: Genome wide association study; HOMA2: Homeostatic model assessment; HOMA2B: Homeostatic model assessment for insulin secretion; HOMA2-IR: Homeostatic model assessment for insulin resistance; LD: Linkage disequilibrium; OGTT: Oral glucose tolerance test; SNP: Single nucleotide polymorphism; T2D: Type 2 diabetes
}

\section{Acknowledgements}

We wish to thank the World Diabetes Foundation for providing a database in Punjab, India and Mr. Raman Gautam for coordinating screening and sampling, Dr. Baldeep and his team from Deep Hospital, Ludhiana, India for providing the infrastructure for the study and the government health authorities of Punjab for supporting the study. We gratefully acknowledge Gabriella Gremsperger, Maria Sterner, Malin Neptin and Jasmina Kravic for 
their technical assistance, sampling and organization of data. Finally, we thank all the participating pregnant women in the study.

\section{Funding}

Funding was received from the World Diabetes Foundation, Denmark, the Danish Strategic Research Council, Novo Nordisk Foundation, the Augustinus Foundation, Center for Physical Activity Research and by Deep Hospital and Ved Nursing Home and Eye Hospital, Ludhiana, India, Sydvästra Skånes Diabetesförening, the Swedish Research Council, Hospital Region of Region Skåne, the Swedish Research Council Networking Grant and the European Research Council.

\section{Availability of data and materials}

All data generated or analysed during this study are included in this published article. Individual level genotyping datasets generated and/or analysed during the current study are not publicly available due being part of other ongoing work but are available from the corresponding author on reasonable request.

\section{Authors' contributions}

GPA, PA, CB and RPB researched data, and reviewed/edited the manuscript. GPA, RT, RPB, and AAV acquired data. RPB, GPA, AAV, RT, LG contributed to study design and reviewed/edited the manuscript. LG and AAV contributed to the discussion and extensively reviewed/edited the manuscript. All authors have read and approved the manuscript. RPB wrote the manuscript. RPB and LG take responsibility for the contents of the article.

\section{Ethics approval and consent to participate}

The study protocol was approved by local Ethical Committees in Punjab and Lund University, complied with the Declaration of Helsinki (2003). All participates gave written informed consent to take part in the present study.

\section{Consent for publication}

This manuscript does not contain individual person's data in any form. Data presented is not identifiable.

\section{Competing interests}

AAV is employed at the Translational Research and Early Clinical Development, Cardiovascular and Metabolic Research, AstraZeneca, Mölndal, Sweden, On behalf of all the authors, Dr. Prasad B has nothing to disclose.

\section{Publisher's Note}

Springer Nature remains neutral with regard to jurisdictional claims in published maps and institutional affiliations.

\section{Author details}

${ }^{1}$ Deep Hospital, Ludhiana, Punjab, India. ${ }^{2}$ Department of Clinical Sciences, Clinical Research Centre, Lund University, Malmö, Sweden. ${ }^{3}$ Department of Endocrinology (Diabetes and Metabolism), Rigshospitalet, Copenhagen, Denmark. ${ }^{4}$ Finnish Institute of Molecular Medicine (FIMM), Helsinki University, Helsinki, Finland. ${ }^{5}$ Cardiovascular and Metabolic Disease (CVMD) Translational Medicine Unit, Early Clinical Development, IMED Biotech Unit, AstraZeneca, Gothenburg, Sweden.

Received: 4 March 2018 Accepted: 25 July 2018

Published online: 08 August 2018

\section{References}

1. Freinkel N. Banting Lecture 1980. Of pregnancy and progeny. Diabetes. 1980;29(12):1023-35

2. Freinkel N. Gestational diabetes 1979: philosophical and practical aspects of a major public health problem. Diabetes Care. 1980;3(3):399-401.

3. National Diabetes Data Group. Classification and diagnosis of diabetes mellitus and other categories of glucose intolerance. Diabetes. 1979;28(12): 1039-57.

4. Engelgau MM, et al. The epidemiology of diabetes and pregnancy in the $U$. S., 1988. Diabetes Care. 1995;18(7):1029-33.

5. Jovanovic L, Pettitt DJ. Gestational diabetes mellitus. JAMA. 2001:286(20): 2516-8.
6. Metzger BE. Summary and recommendations of the third international workshop-conference on gestational diabetes mellitus. Diabetes. 1991; 40(Suppl 2):197-201.

7. Kalra S, Malik S, John M. Gestational diabetes mellitus: a window of opportunity. Indian J Endocrinol Metab. 2011;15(3):149-51.

8. Arora GP, et al. Prevalence and risk factors of gestational diabetes in Punjab, North India: results from a population screening program. Eur J Endocrinol. 2015;173(2):257-67.

9. Kim C, et al. Does frank diabetes in first-degree relatives of a pregnant woman affect the likelihood of her developing gestational diabetes mellitus or nongestational diabetes? Am J Obstet Gynecol. 2009;201(6):576 e1-6.

10. Robitaille J, Grant AM. The genetics of gestational diabetes mellitus: evidence for relationship with type 2 diabetes mellitus. Genet Med. 2008; 10(4):240-50.

11. Buchanan TA, Xiang AH. Gestational diabetes mellitus. J Clin Invest. 2005; 115(3):485-91.

12. Martin $\mathrm{AO}$, et al. Frequency of diabetes mellitus in mothers of probands with gestational diabetes: possible maternal influence on the predisposition to gestational diabetes. Am J Obstet Gynecol. 1985;151(4):471-5.

13. Williams MA, et al. Familial aggregation of type 2 diabetes and chronic hypertension in women with gestational diabetes mellitus. J Reprod Med. 2003;48(12):955-62.

14. Prasad RB, Groop L. Genetics of type 2 diabetes-pitfalls and possibilities. Genes (Basel). 2015;6(1):87-123.

15. Lauenborg J, et al. Common type 2 diabetes risk gene variants associate with gestational diabetes. J Clin Endocrinol Metab. 2009;94(1):145-50.

16. Huopio $\mathrm{H}$, et al. Association of risk variants for type 2 diabetes and hyperglycemia with gestational diabetes. Eur J Endocrinol. 2013;169(3):291-7.

17. Mao H, Li Q, Gao S. Meta-analysis of the relationship between common type 2 diabetes risk gene variants with gestational diabetes mellitus. PLoS One. 2012;7(9):e45882

18. Cho YM, et al. Type 2 diabetes-associated genetic variants discovered in the recent genome-wide association studies are related to gestational diabetes mellitus in the Korean population. Diabetologia. 2009:52(2):253-61.

19. Kwak $\mathrm{SH}$, et al. A genome-wide association study of gestational diabetes mellitus in Korean women. Diabetes. 2012;61(2):531-41.

20. Kim JY, et al. Melatonin receptor 1 B polymorphisms associated with the risk of gestational diabetes mellitus. BMC Med Genet. 2011;12:82.

21. Saxena R, et al. Genome-wide association study identifies a novel locus contributing to type 2 diabetes susceptibility in Sikhs of Punjabi origin from India. Diabetes. 2013;62(5):1746-55.

22. Tabassum $\mathrm{R}$, et al. Genome-wide association study for type 2 diabetes in Indians identifies a new susceptibility locus at 2q21. Diabetes. 2013;62(3): 977-86.

23. Kooner JS, et al. Genome-wide association study in individuals of south Asian ancestry identifies six new type 2 diabetes susceptibility loci. Nat Genet. 2011:43(10):984-9.

24. Radha $\mathrm{V}$, et al. Role of genetic polymorphism peroxisome proliferatoractivated receptor-gamma2 Pro12Ala on ethnic susceptibility to diabetes in south-Asian and Caucasian subjects: evidence for heterogeneity. Diabetes Care. 2006;29(5):1046-51.

25. Abate N, et al. ENPP1/PC-1 K121Q polymorphism and genetic susceptibility to type 2 diabetes. Diabetes. 2005;54(4):1207-13.

26. Kanthimathi $S$, et al. Identification of genetic variants of gestational diabetes in south Indians. Diabetes Technol Ther. 2015:17(7):462-7.

27. Kanthimathi $S$, et al. Association of recently identified type 2 diabetes gene variants with gestational diabetes in Asian Indian population. Mol Gen Genomics. 2017;292(3):585-91.

28. Arora GP, et al. Insulin secretion and action in north Indian women during pregnancy. Diabet Med. 2017;34(10):1477-82. https://doi.org/10.1111/dme. 13428.

29. Levy JC, Matthews DR, Hermans MP. Correct homeostasis model assessment (HOMA) evaluation uses the computer program. Diabetes Care. 1998;21(12): 2191-2.

30. Sokhi J, et al. Association of genetic variants in INS (rs689), INSR (rs1799816) and PP1G.G (rs1799999) with type 2 diabetes (T2D): a case-control study in three ethnic groups from north-West India. Mol Gen Genomics. 2016;291(1): 205-16.

31. Gabriel S, Ziaugra L, Tabbaa D. SNP genotyping using the Sequenom MassARRAY iPLEX platform. Curr Protoc Hum Genet. 2009;60:2-12. Chapter 2: p. Unit 212 
32. Skol AD, et al. Joint analysis is more efficient than replication-based analysis for two-stage genome-wide association studies. Nat Genet. 2006;38(2):209-13.

33. Purcell S, Cherny SS, Sham PC. Genetic power calculator: design of linkage and association genetic mapping studies of complex traits. Bioinformatics. 2003;19(1):149-50.

34. Been LF, et al. A low frequency variant within the GWAS locus of MTNR1B affects fasting glucose concentrations: genetic risk is modulated by obesity. Nutr Metab Cardiovasc Dis. 2012;22(11):944-51.

35. Reich D, et al. Reconstructing Indian population history. Nature. 2009; 461 (7263):489-94.

36. Yajnik CS. Early life origins of insulin resistance and type 2 diabetes in India and other Asian countries. J Nutr. 2004;134(1):205-10.

37. Hodson DJ, et al. ADCY5 couples glucose to insulin secretion in human islets. Diabetes. 2014;63(9):3009-21.

38. Chen $\mathrm{R}$, et al. Type 2 diabetes risk alleles demonstrate extreme directional differentiation among human populations, compared to other diseases. PLoS Genet. 2012;8(4):e1002621.

39. Lin Pl, et al. No gene is an island: the flip-flop phenomenon. Am J Hum Genet. 2007:80(3):531-8.

40. Mancina RM, et al. The COBLL1 C allele is associated with lower serum insulin levels and lower insulin resistance in overweight and obese children. Diabetes Metab Res Rev. 2013;29(5):413-6.

Ready to submit your research? Choose BMC and benefit from:

- fast, convenient online submission

- thorough peer review by experienced researchers in your field

- rapid publication on acceptance

- support for research data, including large and complex data types

- gold Open Access which fosters wider collaboration and increased citations

- maximum visibility for your research: over $100 \mathrm{M}$ website views per year

At BMC, research is always in progress.

Learn more biomedcentral.com/submissions 